\title{
Telomeres are shorter in myocardial infarction patients compared to healthy subjects: correlation with environmental risk factors
}

\author{
Cécilia G. Maubaret • Klelia D. Salpea • Anjly Jain • \\ Jackie A. Cooper • Anders Hamsten • Julie Sanders • \\ Hugh Montgomery • Andrew Neil • Devaki Nair • \\ Steve E. Humphries • \\ HIFMECH consortium, Simon Broome Research \\ Group
}

Received: 6 January 2010 /Revised: 25 February 2010 /Accepted: 11 March 2010/Published online: 11 April 2010

(C) The Author(s) 2010. This article is published with open access at Springerlink.com

\begin{abstract}
Shorter telomeres have been reported in premature myocardial infarction (MI) patients. Our work aimed at confirming the association of shorter telomere with MI in two case-control studies and in familial hypercholesterolemia (FH) patients with coronary heart disease (CHD). The HIFMECH study compared 598 white male patients ( $<60$ years) who survived a first MI and 653 age-matched controls from North and South Europe. Additionally, from the UK, 413 coronary artery bypass graft (CABG) patients and two groups of 367 and 94 FH patients, of whom 145 and 17 respectively had premature CHD, were recruited. Leukocyte telomere length (LTL) was measured using a real-time polymerase chain reaction-based method. In HIFMECH, LTL was significantly shorter in subjects from
\end{abstract}

Electronic supplementary material The online version of this article (doi:10.1007/s00109-010-0624-3) contains supplementary material, which is available to authorized users.

C. G. Maubaret $(\bowtie) \cdot$ K. D. Salpea J J. A. Cooper •

S. E. Humphries

Centre for Cardiovascular Genetics, BHF Laboratories, the Rayne Building, Department of Medicine,

University College London (UCL),

5 University Street,

London WC1E 6JF, UK

e-mail:rmhacm1@ucl.ac.uk

A. Jain · D. Nair

Department of Clinical Biochemistry,

Royal Free Hampstead NHS Trust,

London, UK

A. Hamsten

Atherosclerosis Research Unit, Department of Medicine Solna,

Karolinska Institutet,

Stockholm, Sweden the North (7.99 kb, SD 4.51) compared to the South (8.27 kb, SD 4.14; $p=0.02)$ and in cases (7.85 kb, SD 4.01) compared to controls $(8.04 \mathrm{~kb}, \mathrm{SD} 4.46 ; p=0.04)$. In the CABG study, LTL was significantly shorter $(6.89 \mathrm{~kb}, \mathrm{SD}$ 4.14) compared to the HIFMECH UK controls (7.53, SD $5.29 ; p=0.007)$. In both samples of FH patients, LTL was shorter in those with CHD (overall $8.68 \mathrm{~kb}$, SD 4.65) compared to the non-CHD subjects $(9.23 \mathrm{~kb}, \mathrm{SD} 4.83 ; p=$ 0.012). Apart from a consistent negative correlation with age, LTL was not associated across studies with any measured CHD risk factors. The present data confirms that subjects with CHD have shorter telomeres than controls and extends this to those with monogenic and polygenic forms of CHD.

\author{
J. Sanders \\ Department of Epidemiology and Public Health, UCL, \\ Gower Street Campus, \\ London WC1E 7HN, UK \\ H. Montgomery \\ Institute for Human Health and Performance, \\ Charterhouse Building, UCL Archway Campus, \\ Highgate Hill, \\ London N19 5LW, UK
}

A. Neil

Division Public Health \& Primary Health Care,

University of Oxford,

Oxford OX3 7LF, UK 
Keywords Telomere length · Myocardial infarction . Coronary artery bypass grafting $(\mathrm{CABG})$.

Familial hypercholesterolemia (FH)

\section{Introduction}

Coronary heart disease (CHD) and its main complication, myocardial infarction (MI), are the leading cause of morbidity and mortality in most industrialised nations throughout the world. Approximately one in five deaths in men and one in six deaths in women in UK are caused by CHD (www.heartstats.org). Coronary artery bypass grafting (CABG) is a common option for myocardial revascularization for suitable patients. While in general CHD has a multifactorial origin, in some cases it has a monogenic cause such as familial hypercholesterolemia (FH). FH is an autosomal co-dominant disorder which leads to high levels of low-density lipoprotein (LDL)-cholesterol and, if untreated, to early CHD [1]. Heterozygous FH has been estimated to affect about one in 500 of the British population [2].

Risk of CHD is partly heritable and highly variable between individuals. It is therefore important to predict disease and initiate treatment to prevent events inducing permanent cardiac damage. Since CHD risk is age-related, there is a need for a marker of biological age in evaluating the risk for early onset of CHD, and recent studies have suggested telomere shortening to serve as such [3, 4]. Telomeres are specialised DNA-protein structures at the end of all chromosomes, which preserve chromosome stability and integrity. In normal cells, the DNA replication machinery is unable to completely duplicate the telomeric DNA, and thus telomeres are shortened after each cell division (reviewed by [5]). This may be compensated by the action of the telomerase enzyme in some tissues and/or at certain state in life (reviewed by [6]). On the other hand, oxidative stress accelerates telomere shortening in cells [7], and lowered plasma total antioxidant status correlate with shorter telomere length in diabetics [8]. Telomere length is highly variable at birth and decreases with age (reviewed by [9]). Previous studies have demonstrated an association between telomere length, health and longevity [10]. Shorter leukocyte telomeres have been measured in MI cases [11] and offspring of MI cases [12], type II diabetes [13-15] and other degenerative disease such as dementia and Alzheimer disease [16]; in this last study, mean telomere length was shorter among subjects dying during follow-up than in those surviving.

In the present study, we examined the association of mean leukocyte telomere length (LTL) with CHD in three cohorts of patients: HIFMECH (hypercoagulability and impaired fibrinolytic function mechanisms predisposing to
MI), CABG and FH. HIFMECH is a European multicenter case-control study, which has as its aim the identification of differences in risk markers for MI between subjects living in the North and the South of Europe [17]. CABG is a previously described [18] cohort of patients surviving a first elective CABG surgery. For the first time, LTL is evaluated in $\mathrm{FH}$ patients recruited through lipid clinics in the UK [2, 19]. Moreover, we evaluated the association of LTL with environmental and lifestyle risk factors as well as geographical diversity.

\section{Methods}

Study samples

All studies had been designed and carried out according to the Helsinki declaration and received hospital ethics committee approval. All patients gave written informed consent.

The HIFMECH study compares male survivors of a first MI aged $<60$ years (excluding patients with $\mathrm{FH}$ and insulindependent diabetes mellitus) and population-based individuals of the same age and region recruited from four centres in Europe: Stockholm, Sweden and London, England for the North and Marseille, France and San Giovanni Rotondo, Italy representing the South [17]. In all, a total of 598 post-infarction patients and 653 controls were included in the study. Patients and control subjects were examined in parallel in the early morning after an overnight fast. Post-infarction patients were investigated 3 to 6 months after the acute event. Blood samples were obtained from the antecubital vein after an overnight fast, collected into citrate ( $3.8 \%$ citrate, $0.129 \mathrm{~mol} / \mathrm{L}$ ) and centrifuged at $2,500 \mathrm{~g}$ for $30 \mathrm{~min}$ at $4^{\circ} \mathrm{C}$.

The CABG patients were drawn from the coronary artery surgery inflammation study and are described elsewhere [18]. Briefly, all patients undergoing elective first time CABG at the Middlesex Hospital, London, UK, between October 1999 and September 2000 were invited to participate. Subjects undergoing additional surgical procedures (such as valvular surgery or aneurysmectomy), subjects with evidence of a pre-existing inflammatory state or unstable coronary artery disease and subjects who suffered potentially confounding infective postoperative complications or circulatory failure requiring inotropic support were excluded. Aspirin was omitted routinely 10 days before surgery. Blood samples were drawn preoperatively. These were centrifuged immediately $(3,500 \mathrm{~g}, 10 \mathrm{~min})$, and plasma and cells were separated and frozen at $-20^{\circ} \mathrm{C}$. The $\mathrm{CABG}$ group includes 439 people $(20 \%$ women) having different ethnic origin $(83 \%$ Caucasians, $8 \%$ Asians, $2 \%$ Afro-Caribbean, $2 \%$ of other ethnicity and $5 \%$ of unknown origin). 
Two independent groups of FH patients were studied; the first $\mathrm{FH}$ sample comprises 410 definite $\mathrm{FH}$ adult patients $(47.70 \%$ women) recruited from the Simon Broome (SB) Familial Hyperlipidaemia Register for FH (FH-SH). Definite FH is defined as total cholesterol concentration $>7.5 \mathrm{mmol} / \mathrm{l}$, or LDL-cholesterol concentration $>4.9 \mathrm{mmol} / \mathrm{l}$, plus tendon xanthomas in the patient or a first- or second-degree relative [2]. At recruitment, CAD was documented as described [19] in 104 of the 211 men and in 55 of the 199 women with mean ages of onset of 43.1 and 46.5 years, respectively. Telomere length was measured in a second group of $94 \mathrm{FH}$ patients from the Royal Free Hospital (London) (FH-RF). Mean age was 57.7 years old $(\mathrm{SD}=14.6)$, and out of this group, 17 patients had documented CHD.

\section{Phenotypic variables}

Details on lifestyle factors (i.e. smoking, alcohol consumption and physical activity), personal and family medical history and medication and physiological measurements (i.e. height, weight and blood pressure) were acquired using standardised questionnaires and protocols across all sites and have been described in previous publications $[18,19]$.

\section{Measurement of telomere length}

Leukocyte DNA was extracted by the salting-out method [12]. LTL was measured using a quantitative polymerase chain reaction (PCR)-based method [20], as adapted in our laboratory [12]. The relative telomere length was calculated as the ratio of telomere repeats to single-copy gene (SCG) copies ( $\mathrm{T} / \mathrm{S}$ ratio). For each sample, the quantity of telomere repeats and the quantity of SCG copies were determined in comparison to a reference sample in a telomere and a SCG quantitative PCR, respectively. All PCRs were performed in duplicate on the Rotor-Gene 6000 (Corbett Research Ltd, Cambridge, UK), and the raw data were analysed using the comparative quantification analysis (Rotor-Gene 6000 software, Corbett Research Ltd, Cambridge, UK). The specificity of all amplifications was determined by melting curve analysis. All analyses were processed blinded to case-control status of samples. Using the linear regression line between measures obtained by both the PCR-based method and the conventional terminal restriction fragment (TRF) analysis for the same set of 32 samples, as previously described, the corresponding telomere length in base pairs (bp) was calculated from the T/S ratio measured in each subject [12].

To assess the validity of the method, variability in telomere and SCG PCR amplification efficiency was compared between the three studies. Values were comparable, and the standard deviations overlapped, rendering the $\mathrm{T} / \mathrm{S}$ ratios obtained in different runs comparable. To ascertain that inter-assay variability did not interfere with the results, T/S ratio measurements were repeated for 32 samples from the FH-SB group without CHD and 32 UK controls from the HIFMECH study in two runs including samples from both studies. LTL was longer in the FH group $(9.41 \mathrm{~kb}, \mathrm{SD}=6.20)$ compared to the UK controls $(8.49, \mathrm{SD}=5.10 ; p=0.059)$ for this sub-set of samples, as reported for the total group.

Statistical methods

Statistical analysis was performed using Stata (version 10, StataCorp Texas). Analysis was restricted to Caucasians in order to maintain ethnic homogeneity in the sample. Telomere length, body mass index (BMI), triglycerides, insulin, homocysteine, C-reactive protein and systolic blood pressure were not normally distributed, and thus logtransformed data were used for the analysis, and geometric means (approximate SD) are presented in the tables. For the same reasons, fibrinogen and apoB levels were square root transformed. In Table $1, p$ values comparing cases and controls for different parameters are from conditional logistic regression models in HIFMECH taking account of matching on age and centre. For the FH study, mean values are compared by $t$ test. Correlations were assessed using Pearson partial correlations to allow adjustment for age and other covariates. Because FVIIag values could not be transformed to a normal distribution, Spearman rank correlation was used to test the association between FVIIag and adjusted telomere length. For the association of telomere length with lifestyle parameters, $p$ values were obtained from analysis of covariance models, and the regression estimates were used to obtain adjusted mean values. Adjustment was made for age, centre and exercise in HIFMECH, age in CABG and age and gender in FH.

\section{Results}

General characteristics of the study subjects

Mean LTL was successfully determined in 559 cases and 520 controls of the HIFMECH study, 413 cases of the CABG study and $367 \mathrm{SB}$ and 81 FH-RF patients. The characteristics of these subjects are shown in Table 1, and they did not differ significantly from that of the whole group for any of the parameters. In both the HIFMECH and the FH-SB studies, there were the expected significant differences between cases and controls in risk factors such as BMI, diastolic blood pressure, triglyceride levels, IL6 levels and fibrinogen. In HIFMECH, where plasminogen activator inhibitor (PAI) levels were available, cases had significantly lower PAI levels compared to the controls. 
Table 1 Characteristics of study subjects

\begin{tabular}{|c|c|c|c|c|c|c|c|c|c|c|}
\hline & \multicolumn{3}{|l|}{ HIFMECH } & \multirow[t]{2}{*}{ CABG } & \multicolumn{3}{|l|}{ FH-SB } & \multicolumn{3}{|l|}{ FH-RF } \\
\hline & Controls & Cases & $p$ value & & $-\mathrm{CHD}$ & $+\mathrm{CHD}$ & $p$ value & $-\mathrm{CHD}$ & $+\mathrm{CHD}$ & \\
\hline Number (\% women) & $559(0)$ & $520(0)$ & - & $341(20.8)$ & $222(56.8)$ & $145(33.8)$ & $<0.0001$ & $77(61.3)$ & $17(47.1)$ & 0.28 \\
\hline Age (years) & $51.5(5.5)$ & $51.9(5.4)$ & - & $64.9(92.2)$ & $44.3(13.4)$ & $56.1(10.3)$ & $<0.0001$ & $51.9(14.3)$ & $58.7(14.0)$ & 0.08 \\
\hline BMI $\left(\mathrm{kg} / \mathrm{m}^{2}\right)$ & $26.1(3.2)$ & $27.1(3.3)$ & 0.001 & $28.2(4.5)$ & $23.8(4.0)$ & $25.1(3.4)$ & 0.005 & & & \\
\hline SBP $(\mathrm{mmHg})$ & $127.8(14.4)$ & $127.7(16.9)$ & 0.75 & & $123.7(15.6)$ & $130.6(19.7)$ & 0.0003 & & & \\
\hline DBP (mmHg) & $84.0(8.4)$ & $81.7(10.2)$ & $<0.0001$ & & $77.3(9.1)$ & $79.3(11.3)$ & 0.06 & & & \\
\hline Cholesterol $(\mathrm{mmol} / \mathrm{l})$ & $5.52(0.97)$ & $5.40(1.18)$ & 0.06 & $4.71(1.03)$ & $6.91(1.30)$ & $6.35(1.31)$ & $<0.0001$ & $8.76(1.50)$ & $10.11(2.77)$ & 0.02 \\
\hline Triglyceride $(\mathrm{mmol} / \mathrm{l})$ & $1.44(0.61)$ & $1.88(0.77)$ & $<0.0001$ & & $1.24(0.56)$ & $1.46(0.69)$ & 0.001 & $1.52(0.68)$ & $2.16(1.24)$ & 0.03 \\
\hline LDL (mmol/l) & & & & $2.53(0.88)$ & $4.80(1.28)$ & $4.21(1.23)$ & $<0.0001$ & $6.34(1.48)$ & $7.65(2.68)$ & 0.05 \\
\hline HDL (mmol/l) & & & & $1.23(0.35)$ & $1.38(0.35)$ & $1.28(0.36)$ & 0.01 & $1.47(0.40)$ & $1.10(0.29)$ & 0.005 \\
\hline Insulin (mU/l) & $37.8(24.3)$ & $49.3(34.5)$ & $<0.0001$ & & & & & & & \\
\hline Glucose (mmol/l) & & & & $6.19(2.05)$ & $4.82(0.59)$ & $5.07(0.59)$ & 0.0001 & & & \\
\hline Homocysteine $(\mu \mathrm{mol} / \mathrm{l})$ & $8.27(2.59)$ & $8.55(3.36)$ & 0.03 & & $10.54(3.45)$ & $11.57(3.20)$ & 0.005 & & & \\
\hline CRP (mg/l) & $1.23(1.40)$ & $2.22(2.55)$ & $<0.0001$ & $2.29(2.77)$ & $1.16(1.34)$ & $1.42(1.59)$ & 0.10 & & & \\
\hline IL-6 (pg/ml) & $1.24(0.78)$ & $1.97(1.33)$ & $<0.0001$ & $4.55(3.12)$ & $1.58(1.14)$ & $2.18(1.34)$ & $<0.0001$ & & & \\
\hline Fibrinogen $(\mathrm{g} / \mathrm{L})$ & $3.40(0.69)$ & $3.71(0.92)$ & $<0.0001$ & $3.64(0.77)$ & $2.76(0.78)$ & $3.11(0.99)$ & 0.0002 & & & \\
\hline FVIIag (IU/ml) & $1.17(0.39)$ & $1.12(0.45)$ & 0.14 & & & & & & & \\
\hline PAI (citrate; AU/ml) & $35.1(21.9)$ & $40.4(24.3)$ & 0.001 & & $8.27(6.1)$ & $10.62(7.86)$ & 0.002 & & & \\
\hline PAI (stabilite; AU/ml) & $23.4(18.5)$ & $32.9(24.8)$ & $<0.0001$ & & & & & & & \\
\hline $\begin{array}{l}\text { Unadjusted telomere } \\
\text { length (kbp) }\end{array}$ & $8.04(4.51)$ & $7.90(4.05)$ & 0.34 & $6.85(4.23)$ & $9.82(4.88)$ & $9.04(4.74)$ & 0.001 & $7.53(4.05)$ & $6.70(3.45)$ & 0.03 \\
\hline
\end{tabular}

Unadjusted geometric means (standard deviation) are presented

BMI, triglyceride, insulin, homocysteine, CRP, SBP and homocysteine were log-transformed for tests; fibrinogen level is square root transformed $p$ values for HIFMECH are from conditional logistic regression model taking account of the matching on age and centre

$B M I$ body mass index, $S B P$ systolic blood pressure, $D B P$ diastolic blood pressure, $L D L$ low-density lipoprotein, $H D L$ high-density lipoprotein, $C R P$ C-reactive protein, IL-6 interleukin-6, FVIIag factor VII antigen, PAI plasminogen activator inhibitor

Effects of age, gender, life style and environment on telomere length

We first examined the influence of environmental and life style factors on telomere length. As shown in Fig. 1, LTL correlated negatively with age in the HIFMECH $(r=-0.07$, $p=0.03)$, CABG $(r=-0.18, p=0.0003)$ and FH-SB $(r=$ $-0.16, p<0.0002)$ studies. No significant gender difference was found in the CABG group (0.95 in male vs. 0.93 in female, $p=0.74$ ), whereas in both the FH group, men had significantly shorter telomeres than women $(9.23 \mathrm{~kb}$ in men vs. $9.82 \mathrm{~kb}$ in women, $p=0.01$ for the $\mathrm{SB}$ group and $6.93 \mathrm{~kb}$ in men vs. $7.57 \mathrm{~kb}$ in women, $p=0.02$ for the $\mathrm{RF}$ patients). Therefore, telomere length was adjusted for age in all further analysis and for age and gender in analysis concerning the FH group.

Smoking and alcohol intake did not have a significant effect on telomere length (Table 2). However, it appeared that the less active subjects had longer telomeres in the combined HIFMECH cohort $(p=0.005)$. The cases in HIFMECH had a significantly more sedentary lifestyle than controls $(p<0.0001)$; the level of exercise had a significant effect on telomere length in overall population even after adjustment for case-control status $(p=0.005)$. Nevertheless, no significant association between LTL and

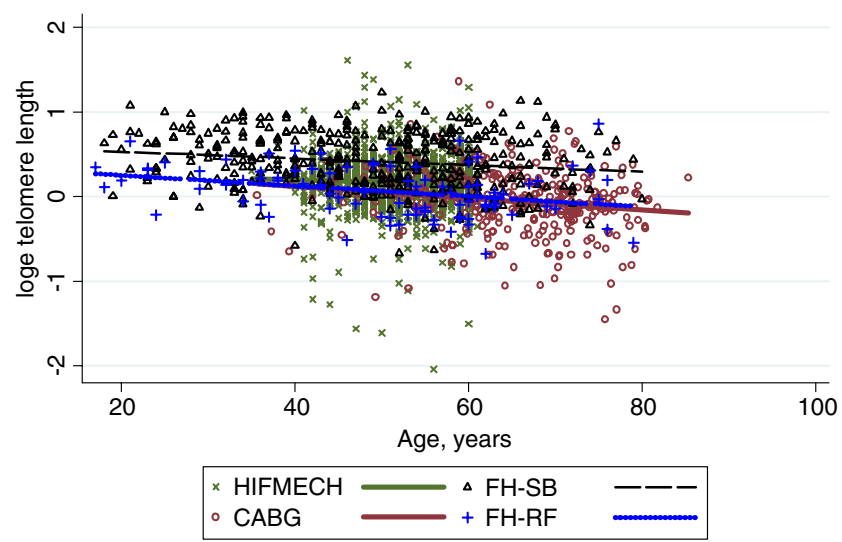

Fig. 1 Decrease of telomere length with age. Telomere length is plotted as loge T/S ratio. $r$ values were $-0.18(p=0.0003),-0.07$ ( $p=$ $0.03)$ and $-0.16(p<0.0002),-0.35(p=0.0002)$ for CABG, HIF$\mathrm{MECH}, \mathrm{FH}-\mathrm{SB}$ and FH-RH, respectively 
Table 2 Lifestyle parameters and telomere length

\begin{tabular}{|c|c|c|c|c|c|c|c|}
\hline & & \multicolumn{2}{|l|}{ HIFMECH } & \multicolumn{2}{|l|}{ CABG } & \multicolumn{2}{|l|}{ FH } \\
\hline & & LTL & $p$ value & LTL & $p$ value & LTL & $p$ value \\
\hline \multirow[t]{3}{*}{ Smoking } & Current & $1.17(0.35) 255$ & \multirow[t]{3}{*}{0.42} & $0.90(0.36) 54$ & \multirow[t]{3}{*}{0.44} & $1.54(0.43) 53$ & \multirow[t]{3}{*}{0.44} \\
\hline & Ex & $1.14(0.36) 518$ & & $0.96(0.34) 212$ & & $1.46(0.50) 125$ & \\
\hline & Never & $1.17(0.41) 306$ & & $0.94(0.34) 75$ & & $1.44(0.47) 189$ & \\
\hline \multirow[t]{3}{*}{ Alcohol } & Yes & $1.16(0.39) 782$ & \multirow[t]{3}{*}{0.74} & & & & \\
\hline & Ex & $1.12(0.34) 61$ & & & & & \\
\hline & Never & $1.17(0.30) 226$ & & & & & \\
\hline \multirow[t]{4}{*}{ Exercise } & Sedentary & $1.17(0.33) 399$ & \multirow[t]{4}{*}{0.005} & & & & \\
\hline & Moderate active & $1.19(0.41) 399$ & & & & & \\
\hline & Active & $1.09(0.36) 183$ & & & & & \\
\hline & Fit & $1.10(0.34) 93$ & & & & & \\
\hline
\end{tabular}

Mean (standard deviation) and number of people in the category are presented. HIFMECH results were adjusted for age and centre. CABG results were age adjusted and FH results age and gender adjusted

fitness level was found in HIFMECH when cases and controls were examined separately. In order to avoid any possible confounding effect in the rest of the analysis in HIFMECH, telomere length was adjusted for exercise level. Age, exercise and case-control status-adjusted telomeres were significantly longer in subjects from the South compared to the North of Europe $(8.27 \mathrm{~kb}, \mathrm{SD}=4.14$ vs.7.99 kb, $\mathrm{SD}=4.51, p=0.02$; Fig. 2), and the difference corresponds to approximately $280 \mathrm{bp}$. Scatter plots of the distribution of the telomere length in North and South are shown in web appendix 1 of the Electronic supplementary material. All further analysis in HIFMECH was also adjusted for region.

Case-control status and telomere length

\section{HIFMECH}

Age-, region- and exercise-adjusted LTL was significantly shorter in CHD cases $(7.85 \mathrm{~kb}, \mathrm{SD}=4.01)$ compared to controls $(8.04 \mathrm{~kb}, \mathrm{SD}=4.46$; Fig. 3). The difference corresponds to approximately $190 \mathrm{bp}$.

\section{$C A B G$}

LTL was significantly shorter in CABG cases $(6.89 \mathrm{~kb}$, $\mathrm{SD}=4.14)$ compared to HIFMECH UK controls $(7.53 \mathrm{~kb}$, $\mathrm{SD}=5.29 ; p=0.007)$ or to all HIFMECH controls $(7.72 \mathrm{~kb}$, $\mathrm{SD}=3.78 ; p<0.0001$ ) (Fig. 3). After 6 years of follow-up in the CABG study, 40 deaths were recorded. Interestingly, subjects who died in these 6 years had marginally shorter telomere $(6.66 \mathrm{~kb}, \mathrm{SD}=4.01)$ compared to those who survived (6.94 kb, $\mathrm{SD}=4.14 ; p=0.28)$.

\section{FH patients}

Amongst the SB patients, age- and gender-adjusted LTL was borderline significantly shorter in those with CHD $(9 \mathrm{~kb}, \mathrm{SD}=4.74)$ compared to those without CHD (9.46 kb, $\mathrm{SD}=4.74 ; p=0.09$; Fig. 3). Additionally, telomere length was compared between 222 FH-SB patients without CHD and 70 UK controls from the HIFMECH study. Adjusted to males aged 49 years, LTL was longer in the FH patients $(9.46 \mathrm{~kb}, \mathrm{SD}=4.74)$ compared to controls $(7.67 \mathrm{~kb}, \mathrm{SD}=$ $5.33 ; p<0.0001)$.
Fig. 2 Comparison of leukocyte telomere length (kilobytes) between North and South in HIFMECH study. Geometric mean of age, exercise and status adjusted telomere length (in kilobytes) and confidence interval
$\mathbf{P}=\mathbf{0 . 0 2}$

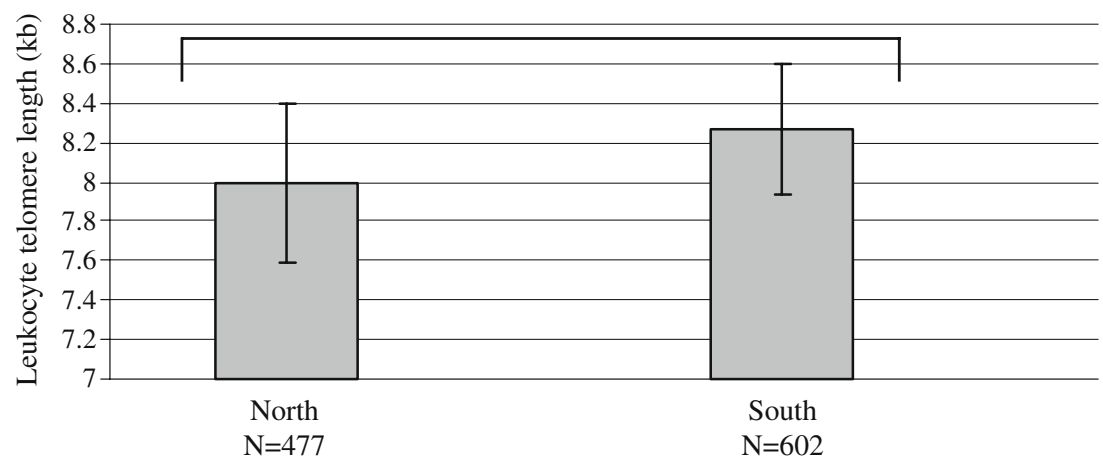


Fig. 3 Comparison of telomere length between cases and controls. Geometric mean of telomere length (in kilobytes) and confidence interval. In HIF$\mathrm{MECH}$, telomere lengths are adjusted by age, region and exercise, in $\mathrm{CABG}$ adjusted to 65 years old and in FH age and gender adjusted

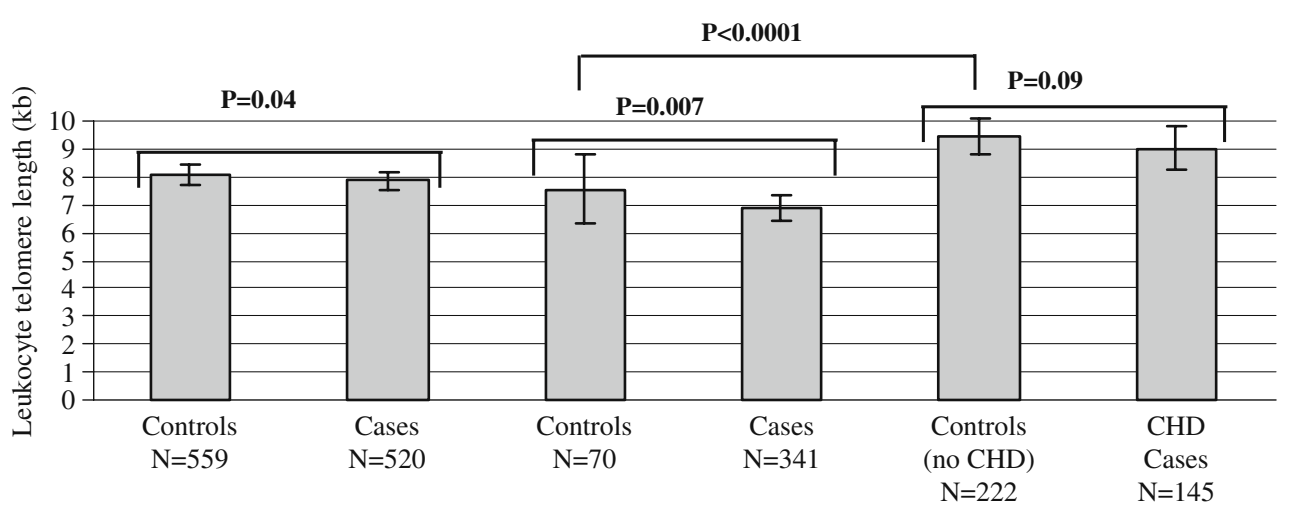

HIFMECH
Amongst the second group of FH patients, age-adjusted LTL was borderline significantly shorter in those with CHD (6.84 kb, SD=3.4) compared to those without $(7.53 \mathrm{~kb}, \mathrm{SD}=$ $4 ; p=0.066)$. However, the difference was not significant when adjusted for age and gender $(p=0.15)$. When combining both cohorts (FH-SB and FH-RF), LTL was shorter in those with CHD (overall $8.68 \mathrm{~kb}$, SD 4.65) compared to the non-CHD subjects (9.23 kb, SD 4.83; $p=0.012)$, confirming the borderline significant association found with the first FH group.

LTL was compared between the FH-RF patients without $\mathrm{CHD}$ to the UK HIFMECH controls. LTL was not longer in the $\mathrm{FH}$ patients $(7.53 \mathrm{~kb}, \mathrm{SD}=4)$ compared to controls (7.67 kb, $\mathrm{SD}=5.33 ; p=0.93)$, suggesting that the difference found previously in the FH-SB group may be a chance effect.

\section{Risk factors, medication and telomere length}

Overall, the association between CHD and shorter LTL is very consistent for the four studies presented here. Given this consistent association, the correlation between telomere length and classical CHD risk factors was examined. As shown in Table 3, in the FH-SB subjects, BMI, triglyceride and fibrinogen levels correlated positively with telomere length $(r=0.26, p<0.0001, r=0.11, p=0.03$ and $r=0.11, p=$ 0.04 , respectively) but these effects were not consistent across studies. In HIFMECH cases, insulin level correlated negatively with telomere length $(r=-0.15, p=0.005)$, Insulin levels were not available in the $\mathrm{CABG}$ and $\mathrm{FH}$ cohorts. Glucose level displayed positive correlation with telomere length in the FH-SB patients $(r=0.14, p=0.006)$ but not in the CABG group $(r=-0.06, p=0.38)$. No association was found between telomere length and diabetes, hypertension or hyperlipidaemia in either the HIFMECH (cases and controls tested separately) and the CABG cohorts. A strong correlation was observed between LTL and IL-6 levels in CABG patients $(r=0.23, p<0.0001)$. Scatter plots for the three stronger associations (glucose in FH, IL-6 in CABG and insulin in HIFMECH cases) are available as web appendix 2 to 4 of the Electronic supplementary material. Mean PAI levels were significantly lower in HIFMECH cases compared to controls, and a weak negative correlation was found between telomere length and stability PAI level $(r=-0.08, p=0.02$ when cases and controls are combined).

Finally, the effect of statin treatment on telomere length in FH patients was assessed. Figure 4 presents the inverse correlation observed between the duration of statin use and telomere length $(r=-0.20, p=0.0004, N=302)$ in the FHSB group. No correlation was found in the second group of FH-RF patients $(r=0.02, p=0.85, N=66)$.

\section{Discussion}

In this study, we report shorter LTL in premature MI male patients, in $\mathrm{CHD}$ patients undergoing a first elective $\mathrm{CABG}$ and in FH patients with early CHD compared to those without CHD. Using the correlation between telomere length and age, the decline in telomere length is approximately 14 bp per year in HIFMECH and 34 bp per year in FH studies. From these values, the difference in mean LTL between CHD cases and controls (190 bp in HIFMECH and $460 \mathrm{bp}$ in $\mathrm{FH}$ ) corresponds to a biological age gap of more than 10 years. MI cases had LTL of healthy subjects who were roughly 15 years older in HIFMECH, and in the FH-SB group, CHD cases had telomeres of 17 years older FH controls. This is in accordance with Brouilette et al. [11] who found that premature MI cases have on average a biological age equivalent to 11.3 years older than controls. Our data, coupled with those of others [11, 21-24], strongly suggest that shorter telomere length is a risk factor for CHD and MI. A recent study by Wilson et al. [25] showed that telomere length in vascular wall cells from abdominal aortas of patients undergoing elective open repair, as well as from normal aortas of cadaveric donors strongly correlates with LTL of the same subjects $(r=0.62, p=$ 0.001). Therefore, overall, the data suggest that MI and CHD are linked to excessive ageing of the vascular wall, 
Table 3 Partial Pearson correlation coefficients of telomere length with classical risk factors

\begin{tabular}{|c|c|c|c|c|c|c|c|c|}
\hline & \multicolumn{4}{|c|}{ HIFMECH } & \multirow{2}{*}{\multicolumn{2}{|c|}{$\frac{\text { CABG }}{\text { Cases }}$}} & \multirow{2}{*}{\multicolumn{2}{|c|}{$\frac{\mathrm{FH}}{\text { Cases }}$}} \\
\hline & \multicolumn{2}{|l|}{ Controls } & \multicolumn{2}{|l|}{ Cases } & & & & \\
\hline & $r$ & $p$ value & $r$ & $p$ value & $r$ & $p$ value & $r$ & $p$ value \\
\hline BMI $\left(\mathrm{kg} / \mathrm{m}^{2}\right)$ & -0.07 & 0.09 & -0.07 & 0.13 & 0.03 & 0.63 & 0.26 & $<0.0001$ \\
\hline SBP $(\mathrm{mmHg})$ & -0.01 & 0.76 & 0.04 & 0.35 & & & -0.06 & 0.29 \\
\hline DBP (mmHg) & -0.02 & 0.60 & -0.02 & 0.73 & & & -0.08 & 0.13 \\
\hline Cholesterol (mmol/l) & -0.02 & 0.61 & -0.03 & 0.56 & -0.01 & 0.86 & -0.03 & 0.54 \\
\hline Triglyceride (mmol/l) & -0.004 & 0.93 & -0.07 & 0.15 & & & 0.11 & 0.03 \\
\hline $\mathrm{LDL}(\mathrm{mmol} / \mathrm{l})$ & & & & & -0.02 & 0.8 & -0.06 & 0.28 \\
\hline HDL (mmol/l) & & & & & -0.02 & 0.8 & -0.03 & 0.54 \\
\hline Insulin $(\mathrm{mU} / \mathrm{l})$ & -0.008 & 0.87 & -0.15 & 0.005 & & & & \\
\hline Glucose (mmol/l) & & & & & -0.06 & 0.38 & 0.14 & 0.006 \\
\hline Homocysteine $(\mu \mathrm{mol} / \mathrm{l})$ & -0.03 & 0.53 & -0.06 & 0.16 & & & 0.02 & 0.77 \\
\hline CRP (mg/l) & -0.01 & 0.77 & 0.003 & 0.95 & 0.12 & 0.03 & 0.05 & 0.34 \\
\hline IL-6 (pg/ml) & 0.02 & 0.75 & -0.06 & 0.25 & 0.23 & $<0.0001$ & 0.07 & 0.16 \\
\hline Fibrinogen $(g / L)$ & 0.002 & 0.97 & -0.06 & 0.22 & 0.07 & 0.22 & 0.11 & 0.04 \\
\hline FVIIag (IU/ml) & -0.04 & 0.37 & -0.07 & 0.11 & & & & \\
\hline PAI (citrate) (AU/ml) & -0.06 & 0.19 & -0.06 & 0.17 & & & 0.02 & 0.74 \\
\hline PAI (stabilite) (AU/ml) & -0.09 & 0.06 & -0.08 & 0.09 & & & & \\
\hline
\end{tabular}

Partial Pearson correlation coefficients $r$ was adjusted for age, centre and exercise in HIFMECH, age in CABG and age and gender in FH and further adjusted for case-control status when cases and controls are pooled

BMI, triglyceride, insulin, homocysteine, CRP, SBP and homocysteine were log-transformed for tests; fibrinogen level is square root transformed $B M I$ body mass index, $S B P$ systolic blood pressure, $D B P$ diastolic blood pressure, $L D L$ low-density lipoprotein, $H D L$ high-density lipoprotein, CRP C-reactive protein, IL-6 interleukin-6, FVIIag factor VII antigen, PAI plasminogen activator inhibitor

which is reflected by shorter telomeres in this tissue, and that LTL can serve as a marker of this process. This raises the possibility, as discussed by others [26], that telomere length could be used to identify individual patients who may be at particularly high risk of CHD, and who may

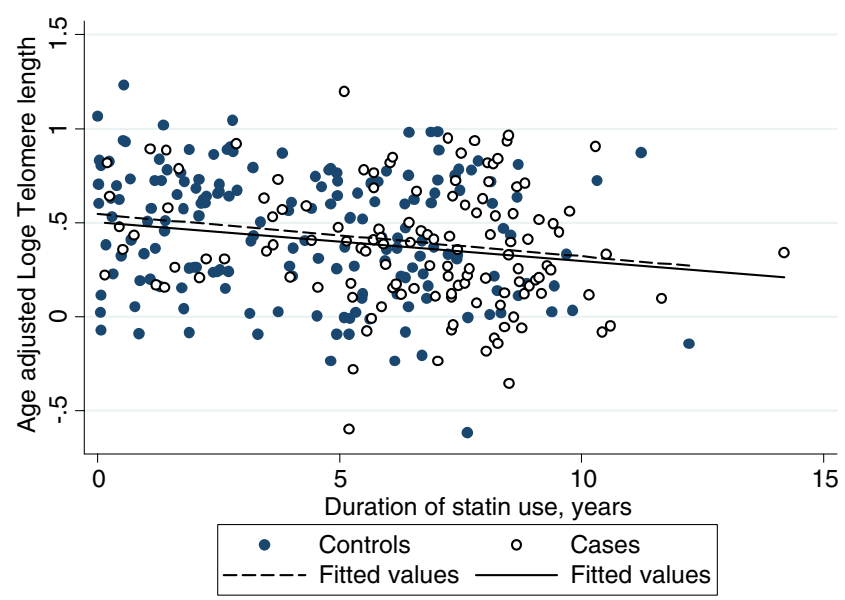

Fig. 4 Correlation between telomere length and duration of statin use in $\mathrm{FH}$ patients. Telomere length is plotted as loge $\mathrm{T} / \mathrm{S}$ ratio. $r$ values were $-0.16(p=0.03, N=175)$ and $-0.18(p=0.05, N=127)$ for CHD and no CHD, respectively benefit from earlier or more intensive statin treatment. However, because telomere length is highly variable at birth and between different ethnicity $[8,9]$, it is not possible to establish a cut-off value in telomere length to distinguish patients who may benefit from early use of medication. Although chronological age is a strong predictor of CHD risk, further research is required to establish the additional relevance of "biological age" in CHD prediction, and to determine absolute values for individual telomere length.

In the HIFMECH sample, telomeres were significantly longer in the South (San Giovanni Rotondo and Marseille) compared to the North of Europe (Stockholm and London). A gradient in CHD risk has been repeatedly reported across Europe (e.g. [27]) and telomere length differences in young healthy male students from middle Europe and UK [12]. In EARSII, telomere length was longer in students from the Middle of Europe $(10.28 \mathrm{~kb})$ compared to the UK $(9.64 \mathrm{~kb})$, the Baltic $(7.95 \mathrm{~kb})$ and the South $(7.62 \mathrm{~kb})$. Comparison between the HIFMECH samples presented here and the EARSII sample is difficult considering the broad definition of the geographical regions in these two studies. Nevertheless, the region considered as South in HIFMECH lies between the geographical regions consid- 
ered as Middle and South in EARSII [12], and the data appear broadly consistent. Although there appears to be some agreement between the well-known gradient of CHD risk across Europe with that seen in telomere length, more data on telomere length in the different European countries is needed to confirm this.

Lifestyle is well known to be one of the stronger predictors of CHD risk, and a modest effect of lifestyle on telomere length has been previously reported. Smoking has been associated with shorter telomeres in controls [28] as well as in lean and obese women [29], while greater physical activity in leisure time has been associated with longer telomeres in healthy twins [30]. In contrast to many published reports, in the four studies presented here, no consistent correlation was found between telomere length and several examined cardiovascular risk factors such as smoking habit, insulin and glucose levels, although in individual studies, some significant associations were observed. However, in any particular study, the absence of a significant correlation can be due to lack of power resulting from the relatively small sample size. Thus, we cannot confirm or refute these reported associations, although in general, they appear to be relatively weak. In the present study, exercise was associated with shorter telomeres in the HIFMECH study, an observation which is in contrast with previously published data. However, this association was not significant when cases and controls were analysed separately. This may suggest that the health status of the study participants had influenced their answers to the lifestyle questionnaire. It seems more likely that the effect of mild exercise on telomere length, if any, is small and therefore difficult to detect, particularly when physical activity is assessed by general questionnaires.

A significant positive association between glucose levels and telomere length was found in the FH subjects of the current study, and this is in accordance with the previous finding of Salpea et al. [12] in the EARSII study $(r=0.21$, $p=0.0001)$. On the other hand, Fitzpatrick et al. [21] reported a weak negative correlation between fasting glucose level and telomere length $(r=-0.0015, p=0.06)$. For the insulin levels, a negative correlation with telomere length was found in the HIFMECH controls, which has also been reported by Aviv et al. [31] in a sample of 18-79 year-old women, representative of the general population $(r=-0.069$, $p=0.023$ ). More work is needed to clarify if any clinically relevant association exists. In vitro work with cell cultures being treated with various glucose concentrations may help us understand this phenomenon. Nevertheless, many studies have shown that subjects with impaired glucose tolerance and type II diabetes have shorter telomeres than controls [13-15]. The association between the presence of diabetes and telomere length was tested in both the HIFMECH and the CABG cohorts, but no significant association was detected. This could possibly be due to the size of the cohort and/or the fact that severity and the complications of the CHD in the cases are masking the effect of diabetes.

This study is the first to evaluate LTL in FH patients. Two independent cohorts of patients were studied. The patients suffering from CHD had roughly 550-bp shorter telomeres than FH patients without CHD. This is in accordance with the results reported for classical polygenic CHD and extends the observation of shorter leukocyte telomeres to this monogenic form of CHD. Interestingly, in the first cohort, the FH patients without CHD had approximately 1,780-bp longer telomeres than healthy controls after adjustment for age and gender. However, the difference was not confirmed in the second group of patients, and the observation of longer telomeres in FH-SB patients free of CHD may be by chance. It is worth mentioning that the mean age in the SB FH patients without CHD was 7 years younger than both the FH-RF patients and the HIFMECH controls. Since telomere length was adjusted for age, this age difference should not be the cause for this difference. However, age may be related to the health status of the patients and/or the medication they have received, which could influence their telomere length. Additionally, the two FH groups were recruited in different centres, which may have caused heterogeneity in the patients' characteristics. Another explanation could be that the SB FH patients without CHD being recruited in this study are the "survivors", and those with shorter telomere length had died earlier. It will be of great interest to evaluate telomere length in more FH patients and if the longer telomeres in FH compared to controls are replicated, then the underlying mechanism should be investigated.

No correlation between total cholesterol levels and telomere length was found in $\mathrm{FH}$ patients or the two other cohorts studied, which is in line with previously published data from our laboratory [12] and from others [11]. Satoh et al. [32] showed recently that in a culture of endothelial progenitor cells (EPC) subject to oxidative stress (tert-butyl hydroperoxide or L-buthionine-[S, R]-sulphoximine), intensive lipid lowering treatment with atorvastatin led to the prevention of EPC telomere shortening. Mahmoudi et al. [33] showed that atorvastatin treatment reduced telomere shortening in vascular smooth muscle cells cultured with tert-butylhydroperoxide, a free radical-generating agent. Considering the protective effect of statins against telomere shortening as shown with these experiments, we reasoned that the longer telomere length in FH-SB patients free of CHD compared to healthy subjects could be a consequence of the prolonged statin treatment that these patients have received. While a proof of this hypothesis would require the availability of follow-up measurements of telomere length 
in subjects under statin or not, the observed significant negative correlation between telomere length and duration of statin use in the FH-SB group renders this explanation less likely. Interestingly, in the prospective WOSCOPS study, Brouilette et al. [22] reported that pravastatin treatment had the greatest benefit for CHD prevention in people in the lowest tertile of telomere length at recruitment compared to those with longest telomeres. Brouilette et al. suggest that the mechanism of this effect may be due to a statin-induced increase in expression of the important telomere capping protein TRF2, which has been reported in cultures of EPC treated with statin [34]. In this case, telomere structure would be stabilised and preventing this way telomere shortening. The direct relevance of this to patients has yet to be confirmed.

It is puzzling that a positive correlation with BMI was found in FH patients, because telomere length is usually negatively correlated with BMI as it was observed for HIFMECH controls and has been shown in other studies [12]. It is relevant that in the FH-SB group, the three risk factors that were significantly correlated with telomere length were also correlated with each other (BMI and triglyceride, $r=0.26, p<0.001$; triglyceride and glucose, $r=$ $0.20, p<0.001$; BMI and glucose, $r=0.20, p<0.001$ ), suggesting that some of the associations observed with telomere length may result from this reason.

In the CABG patients, a weak positive $(r=0.23)$ correlation between pre-operative IL6 levels and telomere length was found, but this association did not persist postoperatively and also was not replicated in the FH group. Other studies [21,35] have reported a negative correlation between telomere length and IL6 levels $(r=-0.408, p=$ $0.08, r=-0.17, p=0.02$ ) in the Cardiovascular Health Study cohort, an observational cohort designed to investigate risk factors for cardiovascular disease in the elderly and multiple myeloma, respectively. The unexpected correlation with IL6 observed in CABG patients before the operation may be due to the condition, the medication and/or changes in the patients' treatment before the operation or to chance.

In conclusion, it is now strongly confirmed that telomere shortening is a marker of normal ageing and CHD along with its complications as MI. Our data in four different casecontrol studies support this concept and include the first data indicating that $\mathrm{FH}$ patients with $\mathrm{CHD}$ have shorter telomeres than FH patients without CHD. Our analysis on the association of CHD risk factors and telomere length confirms the previously observed association with geographical localization of the studied subjects and with glucose levels.

Acknowledgements CM is supported by the Binks Trust. SEH, JC and KS are funded by the British Heart Foundation RG2008/08 and FS/06/053. The HIFMECH study was supported by the European Commission (BMH4-CT96-0272), the Swedish Medical Research Council, the Swedish Heart-Lung Foundation, INSERM and Uni- versité de la Méditerranée (INSERM U626), Foundation pour la Recherche Médicale (FRM) and Programme Hospitalier de Recherche Clinique (PHRC 1996). HIFMECH co-investigators are Anders Hamsten, Steve E. Humphries, Irène Juhan-Vague, Maurizio Margaglione, Giovanni di Minno, John Yudkin and Elena Tremoli. We would like to thank the members of the SB BHF study for the access to patients' samples, as well as Dr. Rossi Naoumova, Prof. Gil Thompson, Dr. Mary Seed, Prof. Paul Durrington, Dr. Paul Miller and Prof. John Betteridge.

Conflicts of interest The authors declare no competing financial interests.

Open Access This article is distributed under the terms of the Creative Commons Attribution Noncommercial License which permits any noncommercial use, distribution, and reproduction in any medium, provided the original author(s) and source are credited.

\section{References}

1. Seed M, Humphries SE, Thorogood M (2009) A commentary on the NICE guideline on identification and management of familial hypercholesterolaemia. PCCJ 2:141-144

2. Neil HAW, Hammond T, Huxley R, Matthews DR, Humphries SE (2000) Extent of underdiagnosis of familial hypercholesterolaemia in routine practice: prospective registry study. Br Med J 321:148

3. Vaziri H, Dragowska W, Allsopp RC, Thomas TE, Harley CB, Lansdorp PM (1994) Evidence for a mitotic clock in human hematopoietic stem-cells-loss of telomeric DNA with age. Proc Natl Acad Sci USA 91:9857-9860

4. Samani NJ, Boultby R, Butler R, Thompson JR, Goodall AH (2001) Telomere shortening in atherosclerosis. Lancet 358:472-473

5. Kappei D, Londono-Vallejo JA (2008) Telomere length inheritance and aging. Mech Ageing Dev 129:17-26

6. Bianchi A, Shore D (2008) How telomerase reaches its end: mechanism of telomerase regulation by the telomeric complex. Mol Cell 31:153-165

7. Kurz DJ, Decary S, Hong Y, Trivier E, Akhmedov A, Erusalimsky JD (2004) Chronic oxidative stress compromises telomere integrity and accelerates the onset of senescence in human endothelial cells. J Cell Sci 117:2417-2426

8. Salpea KD, Talmud PJ, Cooper JA, Maubaret CG, Stephens JW, Abelak K, Humphries SE (2009) Association of telomere length with type 2 diabetes, oxidative stress and $U C P 2$ gene variation. Atherosclerosis. doi:10.1016/j.atherosclerosis.2009. 09.070

9. Aviv A (2008) The epidemiology of human telomeres: faults and promises. J Gerontol 63:979-983

10. Terry DF, Nolan VG, Andersen SL, Perls TT, Cawthon R (2008) Association of longer telomeres with better health in centenarians. J Gerontol 63:809-812

11. Brouilette S, Singh RK, Thompson JR, Goodall AH, Samani NJ (2003) White cell telomere length and risk of premature myocardial infarction. Arterioscler Thromb Vasc Biol 23:842-846

12. Salpea KD, Nicaud V, Tiret L, Talmud PJ, Humphries SE (2008) The association of telomere length with paternal history of premature myocardial infarction in the European Atherosclerosis Research Study II. J Mol Med 86:815-824

13. Sampson MJ, Winterbone MS, Hughes JC, Dozio N, Hughes DA (2006) Monocyte telomere shortening and oxidative DNA damage in type 2 diabetes. Diabetes Care 29:283-289

14. Uziel O, Singer JA, Danicek V, Sahar G, Berkov E, Luchansky M, Fraser A, Ram R, Lahav M (2007) Telomere dynamics in arteries 
and mononuclear cells of diabetic patients: effect of diabetes and of glycemic control. Exp Gerontol 42:971-978

15. Adaikalakoteswari A, Balasubramanyam M, Mohan V (2005) Telomere shortening occurs in Asian Indian Type 2 diabetic patients. Diabetic Med 22:1151-1156

16. Honig LS, Schupf N, Lee JH, Tang MX, Mayeux R (2006) Shorter telomeres are associated with mortality in those with APOE epsilon 4 and dementia. Ann Neurol 60:181-187

17. Juhan-Vague I, Morange PE, Aubert H, Henry M, Aillaud MF, Alessi MC, Samnegard A, Hawe E, Yudkin J, Margaglione M (2002) Plasma thrombin-activatable fibrinolysis inhibitor antigen concentration and genotype in relation to myocardial infarction in the North and South of Europe. Arterioscler Thromb Vasc Biol 22:867-873

18. Brull DJ, Montgomery HE, Sanders J, Dhamrait S, Luong L, Rumley A, Lowe GDO, Humphries SE (2001) Interleukin-6 gene $-174 \mathrm{G}>\mathrm{C}$ and $-572 \mathrm{G}>\mathrm{C}$ promoter polymorphisms are strong predictors of plasma-interleukin-6 levels after coronary artery bypass surgery. Arterioscler Thromb Vasc Biol 21:1458-1463

19. Neil HAW, Seagroatt V, Betteridge DJ, Cooper MB, Durrington PN, Miller JP, Seed M, Naoumova RP, Thompson GR, Huxley R, Humphries SE (2004) Established and emerging coronary risk factors in patients with heterozygous familial hypercholesterolaemia. Heart 90:1431-1437

20. Cawthon RM (2002) Telomere measurement by quantitative PCR. Nucleic Acids Res 30:e47

21. Fitzpatrick AL, Kronmal RA, Gardner JP, Psaty BM, Jenny NS, Tracy RP, Walston J, Kimura M, Aviv A (2007) Leukocyte telomere length and cardiovascular disease in the cardiovascular health study. Am J Epidemiol 165:14-21

22. Brouilette SW, Moore JS, McMahon AD, Thompson JR, Ford I, Shepherd J, Packard CJ, Samani NJ, West of Scotland Coronary Prevention Study Group (2007) Telomere length, risk of coronary heart disease, and statin treatment in the West of Scotland Primary Prevention Study: a nested case-control study. Lancet 369:107-114

23. van der Harst P, van der Steege G, de Boer RA, Voors AA, Hall AS, Mulder MJ, van Gilst WH, van Veldhuisen DJ (2007) Telomere length of circulating leukocytes is decreased in patients with chronic heart failure. J Am Coll Cardiol 49:1459-1464

24. Mukherjee M, Brouilette S, Stevens S, Shetty KR, Samani NJ (2009) Association of shorter telomeres with coronary artery disease in Indian subjects. Heart 95:669-673
25. Wilson WRW, Herbert KE, Mistry Y, Stevens SE, Patel HR, Hastings RA, Thompson MM, Williams B (2008) Blood leucocyte telomere DNA content predicts vascular telomere DNA content in humans with and without vascular disease. Eur Heart J 29:2689-2694

26. Spyridopoulos I, Dimmeler S (2007) Can telomere length predict cardiovascular risk? Lancet 369:81-82

27. Iacoviello L, Arnout J, Buntinx F, Cappuccio FP, Dagnelie PC, de Lorgeril M, Dirckx C, Donati MB, Krogh V, Siani A (2001) Dietary habit profile in European communities with different risk of myocardial infarction: the impact of migration as a model of gene-environment interaction. The IMMIDIET Study. Nutr Metab Cardiovasc Dis 11:122-126

28. McGrath M, Wong JYY, Michaud D, Hunter DJ, De Vivo I (2007) Telomere length, cigarette smoking, and bladder cancer risk in men and women. Cancer Epidemiol Biomarkers Prev 16:815-819

29. Valdes AM, Andrew T, Gardner JP, Kimura M, Oelsner E, Cherkas LF, Aviv A, Spector TD (2005) Obesity, cigarette smoking, and telomere length in women. Lancet 366:662-664

30. Cherkas LF, Hunkin JL, Kato BS, Richards JB, Gardner JP, Surdulescu GL, Kimura M, Lu X, Spector TD, Aviv A (2008) The association between physical activity in leisure time and leukocyte telomere length. Arch Intern Med 168:154-158

31. Aviv A (2006) Telomeres and human somatic fitness. J Gerontol 61:871-873

32. Satoh M, Minami Y, Takahashi $Y$, Tabuchi T, Itoh T, Nakamura M (2009) Effect of intensive lipid-lowering therapy on telomere erosion in endothelial progenitor cells obtained from patients with coronary artery disease. Clin Sci (Lond) 116:827-835

33. Mahmoudi M, Gorenne I, Mercer J, Figg N, Littlewood T, Bennett M (2008) Statins use a novel Nijmegen breakage syndrome-1dependent pathway to accelerate DNA repair in vascular smooth muscle cells. Circ Res 103:717-725

34. Spyridopoulos I, Haendeler J, Urbich C, Brummendorf TH, Oh H, Schneider MD, Zeiher AM, Dimmeler S (2004) Statins enhance migratory capacity by upregulation of the telomere repeat-binding factor TRF2 in endothelial progenitor cells. Circulation 110:3136-3142

35. Wu KD, Orme LM, Shaughnessy J, Jacobson J, Barlogie B, Moore MAS (2003) Telomerase and telomere length in multiple myeloma: correlations with disease heterogeneity, cytogenetic status, and overall survival. Blood 101:4982-4989 